(RESEARCH ARTICLE)

\title{
The interaction effect of abamectin and tramadol on brain neurotransmitters in rats
}

\author{
Kaoud Hussein $1,{ }^{*}$ and Elsaied Ahmed 2 \\ ${ }^{1}$ Department of Veterinary Hygiene and Environmental Pollution, Faculty of Veterinary Medicine, Cairo University, Egypt. \\ 2 Department of Pharmaceuticals Biotechnology, Martin Luther King College, Germany.
}

Publication history: Received on 14 July 2020; revised on 27 July 2020; accepted on 29 July 2020

Article DOI: https://doi.org/10.30574/wjarr.2020.7.1.0262

\begin{abstract}
A single -oral dose of abamectin (10 mg/Kg body weight; LD50) and tramadol dose of $40 \mathrm{mg} \mathrm{kg}^{-1}$ was given orally by gavage to the rats and the brain samples were examined biologically and histopathologically at various times $(0.5,1$, $1.5,2,2.5,3,3.5$ and $24 \mathrm{~h}$ ) after dosing. Results revealed that oxidative stress was noticed, since it provokes a significant in brain due to insecticides administration. A significant reduction in, acetylcholinesterase (AchE), Nor-epinephrine (NE), serotonin (5-HT), epinephrine (E) and dopamine (DA) in treated group as compared to control group (P<0.05). We found that there were neurotoxic effects of pesticides such as abamectin on brain activities, neurotransmitters and alterations in brain tissue. Tramadol, abamectin alone or in combination produced a significant increase in AchE of the brain of treated rats. In normal brain, the activity of acetylcholine esterase, is necessary for the healthy function of the brain, and changes in AchE activity are accompanied by clear signs of neurotoxicity. The central cholinergic neurotransmission in the brain is a crucial factor in cognitive functions including memory and learning.
\end{abstract}

Keywords: Brain Activities; Abamectin; Lethal Dose; Neurotransmitters; Histopathology.

\section{Introduction}

In one hand, environmental exposures of humans to agricultural pesticides that commonly used in agriculture and veterinary medicine and result in chronic neurotoxicity.

On the other hand, some pain killer drugs used in humans that acting in brain may synergist or interact with the pesticides and lead to adverse effect in brain. Several epidemiological analyses have affirmed a link between pesticides and incidence of sporadic Alzheimer's disease (AD). Meanwhile, in vitro and animal models of AD have shed light on potential neuropathological mechanisms [1].

Degenerative diseases of the CNS represent a major disaster to human health. These age-based disorders have become increasingly prevalent, in part due to the increase in the number of elderly people in recent years, such as neurodegenerative diseases such as Alzheimer's disease, Parkinson's disease, Huntington's disease, amyotrophic lateral sclerosis, frontotemporal dementia, and the spinocerebellar ataxias.

These diseases are diverse in their pathophysiology - some of them cause impaired memory, cognitive perception and some other diseases that affect a person's ability to move, speak and breathe.

Abamectin is used as an insecticide and acaricide. It blocks electrical activity in nerve and muscle preparations. The mass of the medium is interrupted by electrical activity in neuromuscular preparations by increasing the membrane conductance to ions of chloride [2].

\footnotetext{
* Corresponding author: Kaoud Hussein
} 
The goal of abamectin includes g-aminobutyric acid (GABA) receptors in the peripheral nervous system. The chloride channel of glutamate gates (GluCl), along with the g-aminobutyric acid chloride channel (GABA) and histamine-gated chloride channel (HisCl), appear to be a target site for avermectin and ivermectin in insects and nematodes [3].

Advanced studies observe that insecticide toxicity may be related to the enhancement production of ROS [4, 5]. Also, the production of ROS has been proposed as a mechanism through which foreign biomechanics and pathologies can produce oxidative stress and cause damage to various tissues [3,6]. Studies in adult rat that describe oxidative stress caused by the pesticide mixture in soft tissues remain rare.

The present study was designed to evaluate the effect of the interaction of abamectin and tramadol on acetyl cholinesterase enzyme and neurotransmitters of brain in male rats.

\section{Material and methods}

\subsection{Chemicals}

\subsubsection{Abamectin}

(ABM) was obtained from the certified reference standard of Abamectin provided from Lab Centre of Pesticides, National Centre of Agricultural Research-Egypt, and was of $>99 \%$ purity. All chemicals used were of analytical grade.

\subsubsection{Tramadol hydrochloride (Td)}

The certified reference of Tramadol (T) was provided from October Pharma Co., Egypt, and was of >99\% purity.

\subsubsection{Animals and treatments}

Nightly six (96) white male rats (Rattus norvegicus) were obtained from the same stock (weight 180-200 g), from the Animal House, Cairo University, Giza, Egypt. The animals were transported to the laboratory where they were placed in a plastic cage and kept in 12-hour light / dark cycle conditions and controlled temperature $\left(25 \pm 2{ }^{\circ} \mathrm{C}\right)$.

They were gotten used to lab conditions for 8 days. All animals had free access to a standard pellet commercial diet and were allowed food and water. Ethical principles in dealing with animals were strictly observed. White rats were randomly divided into four groups of 32 animals each. In addition, 8 male white rats (Rattus norvegicus) were used as a control group. Animal care as well as the experimental procedures was in compliance with guidelines of ethical standards released by Cairo university policy on Animal Care and Use. In order to minimize animal's suffering, we intended only to use the adequate minimal number of animals requested to produce reliable scientific data.

All efforts were made to minimize the number of animals and their suffering in this study through following the guidelines released by Cairo university policy on Animal Care and Use.

\subsubsection{Exposure protocol}

The rats were randomly assigned to 3 groups of 32 animals each and were treated as follows:

\section{Group 1}

The animal group (Tramadol treated group, $N_{1}=32$ ), was divided into 8 sub-groups, each sub-group consisted of 4 rats $\left(n_{1}=4\right)$, received Tramadol at a dose of $40 \mathrm{mg} \mathrm{kg}^{-1}$ administered orally by gavage.

\section{Group 2}

The animal group (Abamectin treated group, $N_{2}=32$ ), was divided into 8 sub-groups, each sub-group consisted of 4 rats $\left(n_{2}=4\right)$ received Abamectin at doses of $10 \mathrm{mg} \mathrm{kg}^{1}$, administered orally by gavage.

\section{Group 3}

The animal group (Tramadol +Abamectin treated group, $N_{3}=32$ ), was divided into 8 sub-groups ,each sub-group consisted of 4 rats $\left(n_{3}=4\right)$ and received Tramadol at a dose of $40 \mathrm{mg} \mathrm{kg}^{-1}$ plus Abamectin at doses of $10 \mathrm{mg} \mathrm{kg}^{-1}$, administered orally by gavage. 
Tramadol and Abamectin were administrated orally by gavages using corn oil.

\section{Control group}

Control group, $\left(\mathrm{N}_{4}=16\right)$, was divided into 8 sub-groups, each sub-group consisted of 2 rats $\left(n_{4}=2\right)$ and received orally (corn oil vehicle; $5 \mathrm{ml} / \mathrm{kg}$ b. wt.).

\subsection{Samples collection}

\subsubsection{Brain samples}

Whole brains were collected at various times $(0.5,1,1.5,2,2.5,3,3.5$ and $24 \mathrm{~h})$ after dosing.

\subsubsection{Blood samples}

Blood samples ( $2 \mathrm{~mL}$ ) were collected into tubes via the supra-orbital or cardiac puncture at constant intervals; half hr., hr1 hr., 1.5 hr., 2 hr. ,2.5 hr,3 hr. 3.5hr and 24 hr., respectively.

\subsection{Determination of Tramadol}

Tramadol concentration was determined by HPLC analysis with an Agilent 1260 HPLC system (USA), with quaternary pump, autosampler injector, thermostat compartment for the column and fluorescence detector (FLD).

\subsection{Determination of Abamectin}

Residues are extracted with acetonitrile. The diluted extract is cleaned up on a $\mathrm{C}_{18}$ solid-phase extraction cartridge. Abamectin residues are derivatized with trifluoroacetic acid and 1-methylimidazole and determined by reversed-phase liquid chromatography with fluorescence detection (excitation: $365 \mathrm{~nm}$ and emission: $470 \mathrm{~nm}$ ) [7].

\subsection{Estimation of biogenic amines [7]}

\subsubsection{Assay of Epinephrine (E), Nor-epinephrine (NE) and Dopamine (DA)}

The aqueous phase was transferred to a tube for assay E, DA and NE fluorescence at excitation wavelengths and emission wavelengths of 380 and $480 \mathrm{~nm}$, respectively. DA fluorescence at excitation and emission wavelengths of 320 and $375 \mathrm{~nm}$, respectively.

\subsubsection{Assay of Serotonin (5-HT)}

The aqueous phase of the spectrophotometer was read at excitation wave lengths and emission wave lengths of 295 and $355 \mathrm{~nm}$, respectively.

\subsubsection{Acetylcholine Esterase (AchE) Enzyme Activity}

The assay of AchE activity was evaluated using a modified method of Ellman et al. [5]. The specific activity of AchE is expressed as $\mu$ moles of ATChI that hydrolyzed mg protein. AC AchE activity was calculated as $\mu$ mol ATChI/ mg BSA $\min ^{-1}$. 


\section{Results and discussion}

Table 1 The mean value ( \pm S.E) of tramadol and abamectin in blood plasma and fecal matter after single oral dose of tramadol ( $40 \mathrm{mg} / \mathrm{Kg}$ body weight) and abamectin $(10 \mathrm{mg} / \mathrm{Kg}$ body weight) of male rats.

\begin{tabular}{lllll}
\hline $\begin{array}{l}\text { Time } \\
\text { hr. }\end{array}$ & Blood plasma & & Faecal matter & \\
Param & $\begin{array}{l}\text { Tramadol } \\
\boldsymbol{\mu g} \backslash \mathbf{m l}\end{array}$ & $\begin{array}{l}\text { Abamectin } \\
\boldsymbol{\mu g} \backslash \mathbf{m l}\end{array}$ & Tramadol $\boldsymbol{\mu g} \backslash \mathbf{g}$ & Abamectin $\boldsymbol{\mu g} \backslash \mathbf{g}$ \\
\hline 0.5 & $4.81 \pm 0.52$ & $0.04 \pm 0.0092$ & $12.417 \pm 1.2$ & 0.0 \\
1 & $4.733 \pm 0.40$ & $0.062 \pm 0.008$ & $11.97 \pm 0.30$ & $0.177 \pm 0.003$ \\
1.5 & $4.252 \pm 0.08$ & $0.0715 \pm 0.004$ & $11.64 \pm 0.40$ & $0.645 \pm 0.004$ \\
2 & $3.64 \pm 0.68$ & $0.1572 \pm 0.002$ & $11.00 \pm 0.22$ & $0.373 \pm 0.002$ \\
2.5 & $3.21 \pm 0.088$ & $0.166 \pm 0.004$ & $10.305 \pm 0.30$ & $0.382 \pm 0.003$ \\
3 & $3.17 \pm 0.092$ & $0.1566 \pm 0.002$ & $10.905 \pm 0.42$ & 0.0 \\
3.5 & $1.50 \pm 0.084$ & $1.104 \pm 0.082$ & $9.12 \pm 0.88$ & 0.0 \\
24 & $0.45 \pm 0.008$ & $0.04 \pm 0.002$ & $2.50 \pm 0.22$ & 0.0 \\
\hline
\end{tabular}
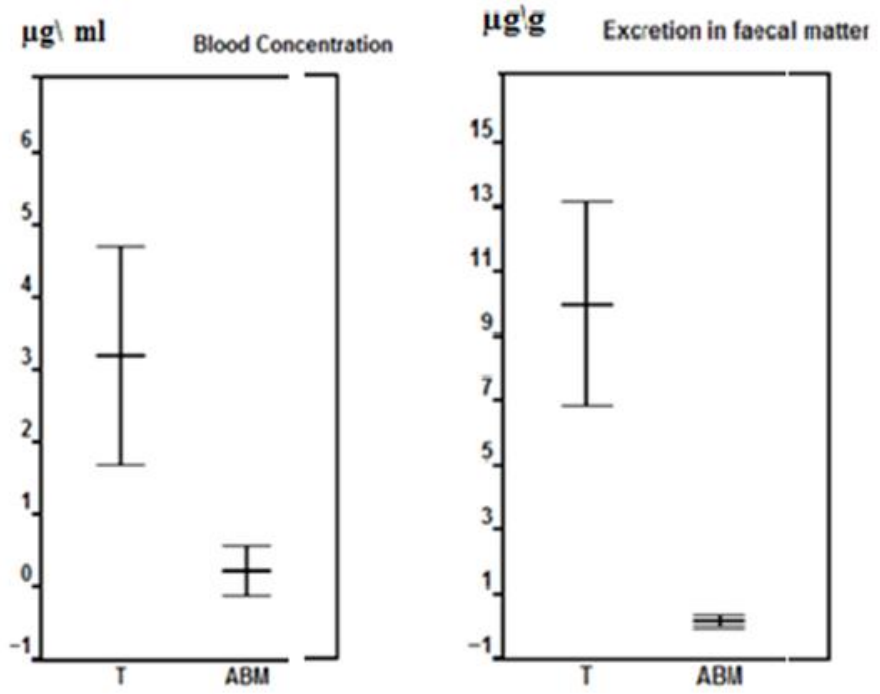

ANOVA-One way-Significant $P>0.05$

ANOVA-One way-Significant $P>0.05$

Figure 1 The mean value $( \pm$ S.E) of tramadol and abamectin in blood plasma and fecal matter after single oral dose of tramadol ( $40 \mathrm{mg} / \mathrm{Kg}$ body weight) and abamectin $(10 \mathrm{mg} / \mathrm{Kg}$ body weight) of male rats. 
Table 2 The mean value $( \pm$ S.E) of tramadol and abamectin in combination in blood plasma and fecal matter after single oral dose of tramadol (40 mg/ Kg body weight) and abamectin (10 mg/Kg body weight) of male rats.

\begin{tabular}{|c|c|c|c|c|}
\hline \multirow{3}{*}{$\begin{array}{l}\text { Time } \\
\text { hr. } \\
\text { Param }\end{array}$} & \multicolumn{4}{|c|}{ In combination } \\
\hline & \multirow{2}{*}{$\begin{array}{l}\text { Blood } \\
\text { Tramadol } \\
\mu \mathrm{g} \backslash \mathrm{ml}\end{array}$} & \multicolumn{3}{|c|}{ Faecal } \\
\hline & & $\begin{array}{l}\text { Abamectin } \\
\mu \mathrm{g} \backslash \mathrm{ml}\end{array}$ & $\begin{array}{l}\text { Tramadol } \\
\mu \mathrm{g} \backslash \mathrm{g}\end{array}$ & $\begin{array}{l}\text { Abamectin } \\
\mu \mathrm{g} \backslash \mathrm{g}\end{array}$ \\
\hline 0.5 & $2.958 \pm 0.08$ & $0.0438 \pm 0.002$ & $8.212 \pm 0.88$ & 0.0 \\
\hline 1 & 2.682 & $0.0824 \pm 0.002$ & $4.944 \pm 0.44$ & 0.0 \\
\hline 1.5 & $2.811 \pm 0.086$ & 0.1074 & $7.301 \pm 0.98$ & 0.0 \\
\hline 2 & $2.904 \pm 0.088$ & $0.0447 \pm 0.012$ & $4.056 \pm 0.99$ & 0.0 \\
\hline 2.5 & $2.899 \pm 0.13$ & $0.0336 \pm 0.008$ & $5.114 \pm 0.88$ & 0.0 \\
\hline 3 & $2.830 \pm 0.089$ & 0.0 & 0.0 & 0.0 \\
\hline 3.5 & $2.931 \pm 0.18$ & $0.874 \pm 0.042$ & $0.874 \pm 0.088$ & 0.0 \\
\hline 24 & $2.957 \pm 0.08$ & $0.0656 \pm 0.002$ & $0.0656 \pm 0.008$ & 0.0 \\
\hline
\end{tabular}

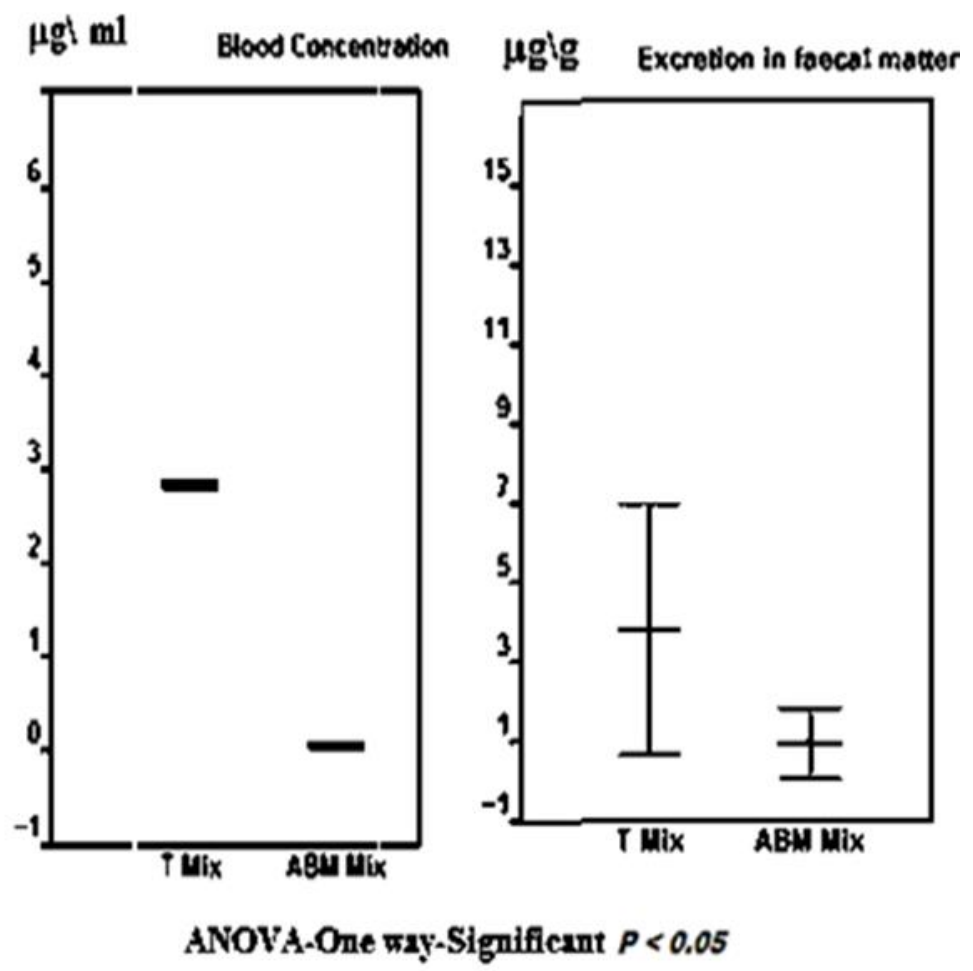

Figure 2 The mean value $( \pm$ S.E) of tramadol and abamectin in combination in blood plasma and fecal matter after single oral dose of tramadol (40 mg/ Kg body weight) and abamectin (10 mg/Kg body weight) of male rats.

\subsection{The interaction between tramadol and abamectin in blood plasma}

We noticed that, the levels of tramadol and ABM were decreased in blood and the tramadol concentration was maintained to constant levels $(2.9 \mu \mathrm{g} \backslash \mathrm{ml})$ during all the intervals of measurement (fig. 2 and table 2). 


\subsection{The interaction between tramadol and abamectin in faces}

We also, noticed that, the levels of tramadol excretion in fecal matter were significantly $(\mathrm{p}<0.05)$ decreased while there was no excretion for ABM in fecal matter during all the intervals of measurement (fig. 2 and table 2).

\subsection{Neurotransmitters}

\subsubsection{Nor-epinephrine}

NE levels were significantly higher $(\mathrm{P}<0.05)$ in the tramadol-treated group $[9,10]$ and significantly lower $(\mathrm{P}<0.05)$ in the abamectin- treated group as compared to the control. Tramadol, on the other hand, minimize the reducing effect of abamectin. Tramadol prevents the uptake of epinephrine and serotonin for its analgesic actions. The change in serotonin levels is responsible for the observed effects of elevation and mood observed by tramadol (fig.3).

This can also be explained by the reversal of depression (caused by low levels of serotonin in the brain) when taking tramadol, or the appearance of depressive symptoms upon its withdrawal.

The norepinephrine carrier (NET) plays a pivotal role in terminating noradrenergic signals and preserving norepinephrine (NE) through the re-uptake process [11].

\subsubsection{Epinephrine (E)}

E levels were significantly $(\mathrm{P}<0.05)$ higher in tramadol-treated group and significantly $(\mathrm{P}<0.05)$ lower in abamectintreated group as compared to control group. On the other hand, tramadol antagonized the reducing effect of abamectin (fig.3). Epinephrine activity in the brain decreased after the administration of abamectin in rat groups, revealed a significant decrease in the mean of epinephrine activities, but increased significantly by tramadol.
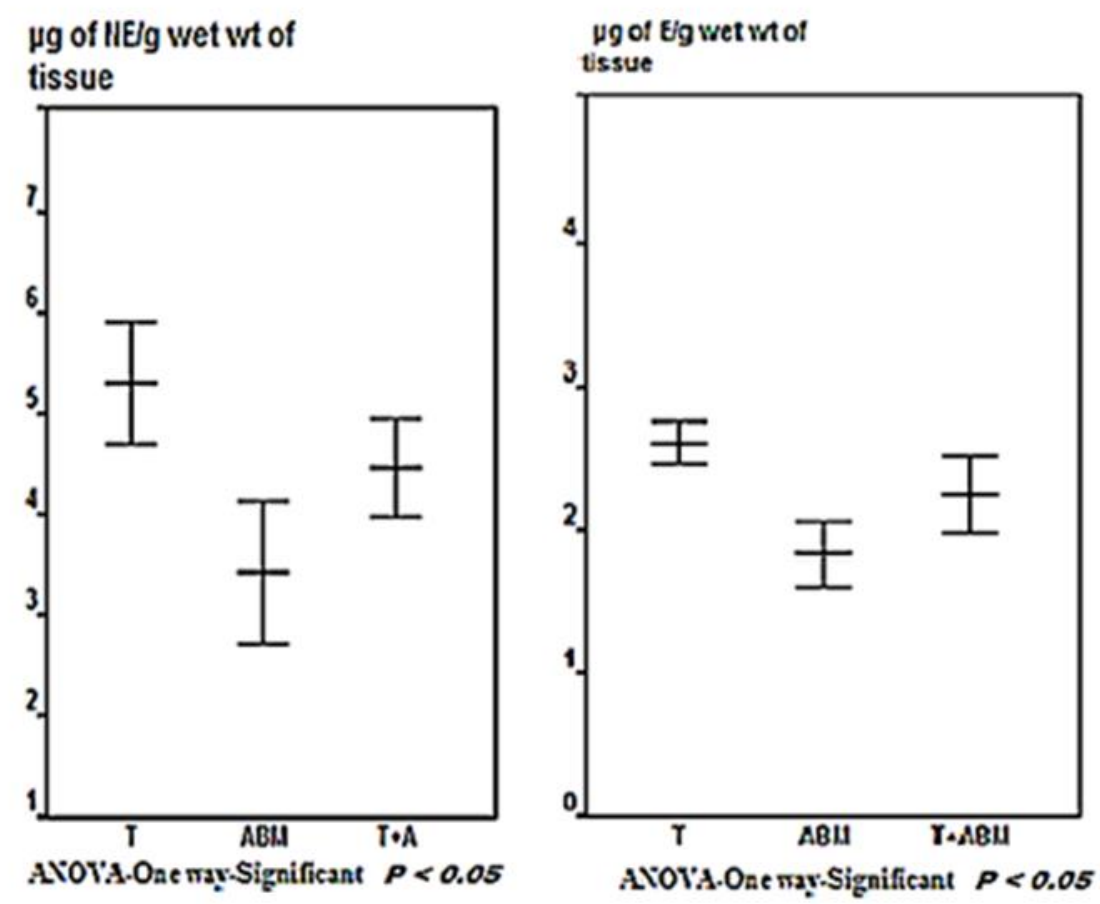

Figure 3 The effect of the interaction between tramadol and abamectin in combination (T+A) after single oral dose of tramadol (40mg/ Kg body weight) and abamectin (10mg / Kg body weight) on nor-epinephrine and epinephrine in male rats.

\subsubsection{Dopamine (DA)}

DA levels were significantly $(\mathrm{P}<0.05)$ higher in tramadol-treated group and significantly $(\mathrm{P}<0.05)$ lower in abamectintreated group as compared to control group (Fig.4). On the other hand, tramadol antagonized the reducing effect of abamectin. Tramadol dose-dependently increased extracellular serotonin and NE levels in the ventral hippocampus of freely moving rats, and was found to affect the uptake and release of NE and DA in hypothalamus [9,10] appears to be 
more active in spinal cord, lower brainstem and CB, whereas DA is often the more effective agent in various parts of the forebrain. DA is considered in great detail when dealing with areas such as striatum, where it is present in particularly large amounts, and is believed to have an important transmitter role [4].

Dopamine activity in the brain decreased after the administration of abamectin in rat groups, revealed a significant decrease in the mean of epinephrine activities, but increased significantly by tramadol. DA content was decreased in the cerebral cortex, midbrain and brainstem regions, whereas it was increased in the cerebellum and thalamus/hypothalamus Tramadol enhances DA turnover via an opioid mechanism. The interaction of Tramadol with the noradrenergic and serotonergic neurotransmission closely resembled that of NA and 5-HT uptake inhibitors.

\subsubsection{Serotonin}

Serotonin levels $(\mathrm{P}<0.05)$ were significantly higher in the tramadol-treated group and significantly lower $(\mathrm{P}<0.05)$ in the abamectin -treated group compared to the control group (Fig.4). Tramadol, on the other hand, is prevent the reducing effect of abamectin on secretion of brain tissue. Tramadol prevents the uptake of serotonin for its analgesic actions. The change in serotonin levels is responsible for the observed effects of elevation and mood observed by tramadol.

This can also be explained by the reversal of depression (caused by low levels of serotonin in the brain) when taking tramadol, or the appearance of depressive symptoms upon withdrawal. Avermectin BIA is a potent inhibitor of the highly affinity uptake system, and its effect is blocked by picrotoxin \{High-affinity uptake of $\left[{ }^{3} \mathrm{H}\right]$ serotonin $\}$ is inhibited by imipramine and amitriptyline). Abamectin promotes the reuptake of serotonin and epinephrine [13].

\subsubsection{AchE}

Tramadol alone, ABM alone or in combination produced a significant increase in Ache of the brain of treated rats (Fig.6). In normal brain, the activity of acetylcholine esterase, is necessary for the healthy function of the brain, and changes in AchE activity are accompanied by clear signs of neurotoxicity. Therefore, this parameter can be used as an indicator of neurotoxicity in animals and humans [14]. The central cholinergic neurotransmission in the brain is a crucial factor in cognitive functions including memory and learning [15,16,5].In contrary with Sahitya et al., [17], they stated that the enzyme activities showed a decrease in all areas of the brain following administration of single dose of ab and abamectin by $24 \mathrm{~h}$ they returned more or less to the respective control levels.

Tramadol is an opioid used as an analgesic to treat moderate to severe pain. It is believed to work by modifying GABAergic, noradrenergic and serotonergic systems. Tramadol and its M1 metabolite were found to bind $\mu$-opioid receptors, so exerting its effect on GABAergic transmission, inhibit 5-HT and noradrenalin reuptake.

The serotonergic modulating properties of tramadol means that it has the ability to interact with other serotonergic agents. There is an increased risk of serotonin syndrome when tramadol is taken in combination with reuptake inhibitors, agents that enhance the effect of 5-HT or 5-HT agonists. Research focused on the aminergic system with reference to the effects of tramadol on its antidepressant effects with reference to uptake and release of NE, 5-HT, DA, along with the potential of dependence and metabolism.

$\mathrm{GABA}_{\mathrm{A}}$ receptors (distributed mainly in the brain) were not affected by tramadol or M1 metabolite at clinically relevant concentrations, but were significantly inhibited only at a large concentration, $(100 \mu \mathrm{M})$. GABAA receptors [18]. Both GABA-gated and GABA-insensitive chloride channels (in mouse brain preparations) may be toxicologically significant targets for the action of avermectins [19]. 


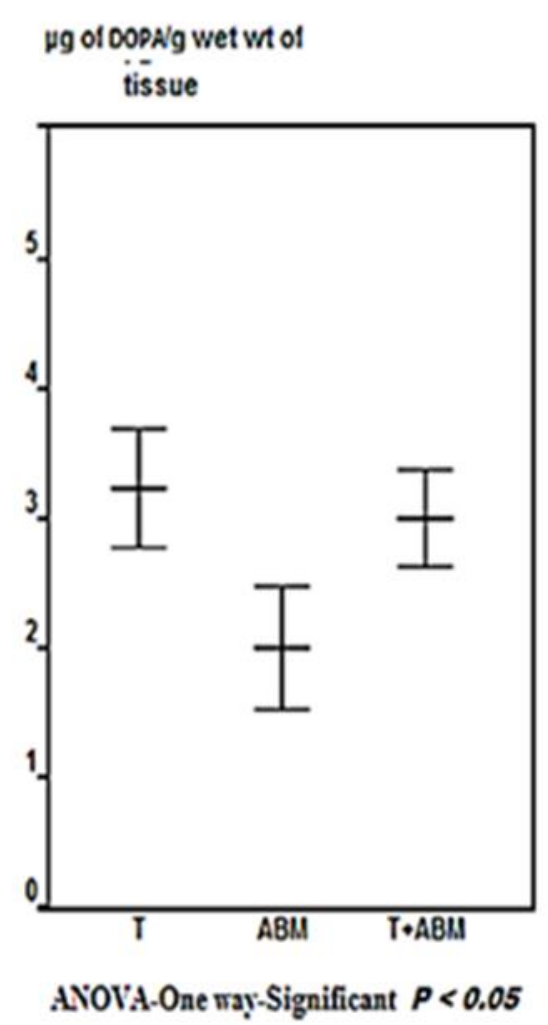

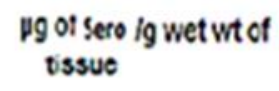

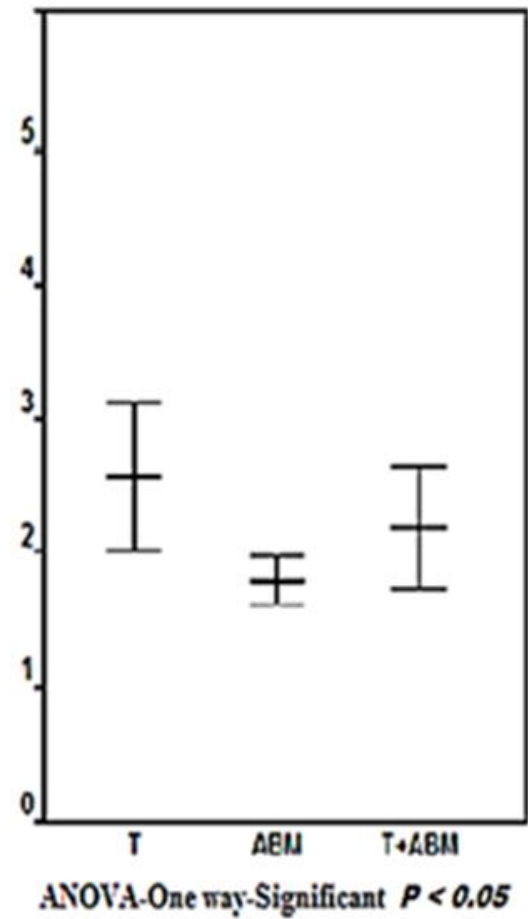

Figure 4 The effect of the interaction between tramadol and abamectin in combination (T+ABM) after single oral dose of tramadol (40mg/ Kg body weight) and abamectin (10 mg/Kg body weight) on dopamine and serotonin in male rats.

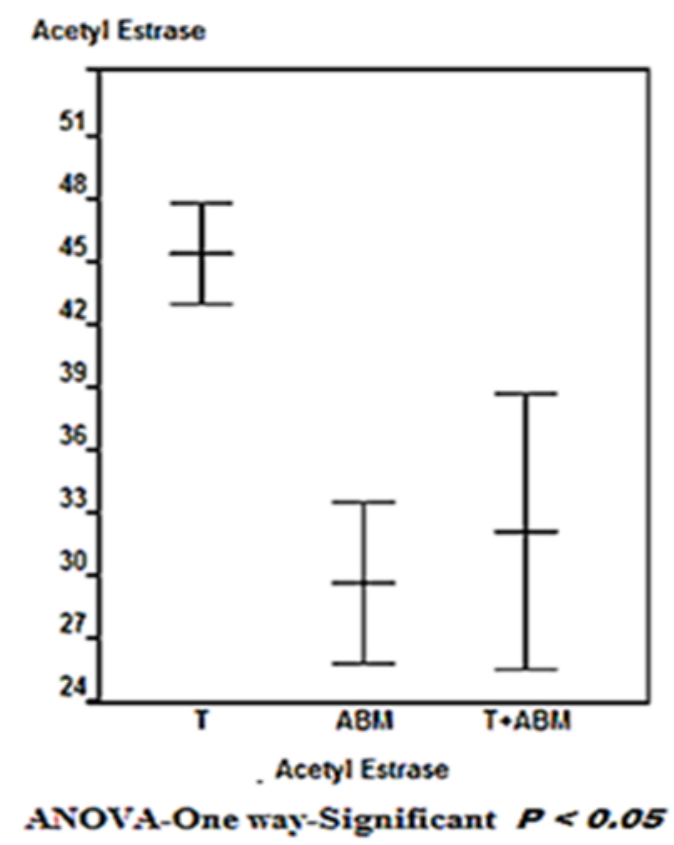

Figure 5 The effect of the interaction between tramadol and abamectin in combination (T+ABM) after single oral dose of tramadol (40 mg/ Kg body weight) and abamectin (10 mg / Kg body weight) on the activity of acetylcholine esterase in male rats. 


\section{Conclusion}

We found that there were neurotoxic effects of pesticides such as abamectin on brain activities, neurotransmitters and alterations in brain tissue. Further pesticide studies are also needed and increased interest in decoding the effects of different types of pesticides. New information is required to investigate the relative importance of exposure and the role of moderate exposure without poisoning. Finally, it will be important to clarify the relationship between neurotoxicity associated with pesticides and neurodegenerative diseases.

\section{Compliance with ethical standards}

\section{Acknowledgments}

This research was sponsored by Department of Animal Hygiene and Environmental Pollution (Faculty of Veterinary Medicine, Cairo University). We thank Dr. Farag Mahmoud Malhat Central Laboratory of Pesticides, Centre of Agricultural Research, Egypt.

\section{Disclosure of conflict of interest}

The authors declare that they have no competing interests.

\section{Statement of ethical approval}

All efforts were made to minimize the number of animals and their suffering in this study through following the guidelines released by Cairo university policy on Animal Care and Use.

\section{References}

[1] Tang BL. (2020). Neuropathological Mechanisms Associated with Pesticides in Alzheimer's Disease. Toxics, 8(2), 21.

[2] McCavera S, Walsh TK and Wolstenholme AL. (2007). Nematode ligand-gated chloride channels: an appraisal of their involvement in macrocyclic lactone resistance and prospects for developing molecular markers. Parasitology, 134, 1111-1121.

[3] Clark j, Scott M, Campos F and Bloomquist JR. (1995). Resistance to aver-mectins: extent, mechanisms, and management implications. Annu Rev Entomol, 40, 1-30.

[4] Bosse KE, Maina FK, Birbeck JA, France MM, Roberts JJ and Colombo ML. (2011). Aberrant striatal dopamine transmitter dynamics in brain-derived neurotrophic factor-deficient mice, J Neurochem, 120(3), 385-95.

[5] Campos C, Rocha NB, Vieira RT, Rocha SA, Telles-Correia D and Paes F. (2016). Treatment of cognitive deficits in Alzheimer's disease: a psychopharmacological review. Psychiatr Danub , 28(1), 2-12.

[6] Ellman GL, Courtney KD, Andres V and Featherstone RM. (1961). A new and rapid colorimetric determination of acetylcholinesterase activity. Biochem. Pharmacol, 7, 88-95.

[7] Diserens H and Henzelin M. (1999). Determination of abamectin residues in fruits and vegetables by highperformance liquid chromatography. Journal of Chromatography, 833(1), 12, 13-18.

[8] Ezzeldin E, Wafaa A HS, El-Nahhas T, Soudi AMM and Shahat AA. (2014). Biochemical and Neurotransmitters Changes Associated with Tramadol in Streptozotocin-Induced Diabetes in Rats. BioMed Research International Volume, Article ID 238780.

[9] Nelson EM and Philbrick AM. (2012). Avoiding Serotonin Syndrome: The nature of the interaction between tramadol and selective serotonin reuptake inhibitors. Annals of Pharmacotherapeutics, 46(12), 1712-6.

[10] Bloms-Funke P, Dremencov E, Cremers TIFH and Tzschentke TM. (2011). Tramadol increases extracellular levels of serotonin and noradrenalin as measured by in vivo microdialysis in the ventral hippocampus of freely moving rats. Neurosci Lett., 490(3), 191-5.

[11] Gomez-Merino D, Béquet F, Berthelot M, Chennaoui M and Guezennec CY. (2001). Site-dependent effects of an acute intensive exercise on extracellular 5-HT and 5-HIAA levels in rat brain. Neurosci Lett, 30, 301(2), 143-6. 
[12] Frink MC, Hennies HH, Englberger W, Haurand M and Wilffert B. (1996). Influence of tramadol on neurotransmitter systems of the rat brain. Arzneim Forsch, 46(11), 1029-36.

[13] Benson GJ. (2005). Physiologic effects of pharmacological agents. Veterinary Clinical Services, Anesthesia and Analgesia. Yale Animal Resource Center.

[14] Milatovic D, Gupta RC and Aschner M. (2006). Anticholinesterase Toxicity and Oxidative Stress. The Scientific World Journal, 6, 295-310

[15] Schliebs R and Arendt T. (2011). The cholinergic system in aging and neuronal degeneration. Behav Brain Res, 221(2), 555-63.

[16] Tan CC, Yu JT, Wang HF, Tan MS, Meng XF and Wang C. (2014). Efficacy and safety of donepezil, galantamine, rivastigmine, and memantine for the treatment of Alzheimer's disease: a systematic review and meta-analysis. J Alzheimers Dis, 41(2), 615-31.

[17] Sahitya Chetan P, Saritha P, Sangeetha LAR, Visweswari G, Murali Mohan P and Rajendra W. (2009). Safety of tramadol - changes in the levels of microsomal cyp450 in rat brain without nociception. Pharmacology online, 2, 699-707.

[18] Yamakura T, Bertaccini E, Trudell JR and Harris RA. (2001). Anesthetics and ion channels: molecular models and sites of action. Annu Rev Pharmacol Toxicol, 41, 23-51.

[19] Payne GT, et al. (1991). Activation of gamma-aminobutyric acid insensitive chloride channels in mouse brain synaptic vesicles by avermectin B1a. J Biochem Toxicol. , Winter, 6(4), 283-92.

\section{How to cite this article}

Kaoud H and Elsaied A. (2020). The interaction effect of abamectin and tramadol on brain neurotransmitters in rats. World Journal of Advanced Research and Reviews, 7(1), 263-272. 